\title{
Alemtuzumab: evidence for its potential in relapsing-remitting multiple sclerosis
}

This article was published in the following Dove Press journal:

Drug Design, Development and Therapy

5 March 2013

Number of times this article has been viewed

\author{
J William L Brown \\ Alasdair J Coles \\ Department of Clinical \\ Neurosciences, University \\ of Cambridge, Cambridge, UK
}

Correspondence: Alasdair J Coles

Department of Clinical

Neurosciences, University

of Cambridge, Box 165,

Addenbrooke's Hospital,

Cambridge CB2 0QQ, UK

$\mathrm{Tel}+44$ I22 32। 675।

Fax +44 I22333 694I

Email ajc1020@medschl.cam.ac.uk

\begin{abstract}
Alemtuzumab (previously known as Campath ${ }^{\circledR}$ ) is a humanized monoclonal antibody directed against the CD52 antigen on mature lymphocytes that results in lymphopenia and subsequent modification of the immune repertoire. Here we explore evidence for its efficacy and safety in relapsing-remitting multiple sclerosis. One Phase II and two Phase III trials of alemtuzumab versus active comparator (interferon beta-1a) have been reported. Two of these rater-blinded randomized studies assessed clinical and radiological outcomes in treatment-naïve patients; one explored patients who had relapsed despite first-line therapy. Compared to interferon beta-1a, alemtuzumab reduced the relapse rate by $49 \%-74 \%(P<0.0001)$, and in two studies it reduced the risk of sustained disability accumulation by $42 \%-71 \%(P<0.01)$. In one study (Comparison of Alemtuzumab and Rebif Efficacy in Multiple Sclerosis; CARE-MS1), there was no significant difference compared to interferon, perhaps reflecting the surprisingly low frequency of disability events in the comparator group. After alemtuzumab, the Expanded Disability Status Scale score improved by $0.14-1.2$ points, culminating in a net advantage with alemtuzumab of 0.41-0.77 points over interferon in the CAMMS223 and CARE-MS2 trials (both $P<0.001$ ). Radiological markers of new lesion formation and brain atrophy following alemtuzumab were significantly improved when compared to interferon in all studies. Adverse events were more common following alemtuzumab than interferon beta-1a (7.2-8.66 versus 4.9-5.7 events per person-year). While infusion reactions are the most common, autoimmunity is the most concerning; within Phase III studies, thyroid disorders (17\%-18\% versus 5\%-6\%) and immune thrombocytopenic purpura ( $1 \%$ versus $0 \%$ ) were reported in patients taking alemtuzumab and interferon beta-1a, respectively. All patients responded to conventional therapy. One patient taking alemtuzumab in the Phase II study suffered a fatal intracranial hemorrhage following immune thrombocytopenic purpura, heralding assiduous monitoring of all patients thereafter. Alemtuzumab has been submitted for licensing in relapsing-remitting multiple sclerosis in the United States and Europe.
\end{abstract}

Keywords: alemtuzumab, Campath, efficacy, relapsing-remitting multiple sclerosis

\section{Introduction}

Multiple sclerosis (MS) is the leading nontraumatic cause of disability in the young, with a total lifetime cost of US $\$ 1.2$ million per patient. ${ }^{1}$ A total of $85 \%-90 \%$ of patients present with a relapsing-remitting form of the disease (RRMS) with resolution of symptoms between flares; most will ultimately evolve into secondary progressive disease with steadily accumulating disability. The disease is pathologically characterized by immune-mediated demyelination and axonal damage. Although previously thought to be CD4+ T-helper lymphocyte-driven, a growing body of evidence suggests 
a complicated (and incompletely understood) interaction of such cells with CD8+ T-cells, B-cells, and the innate immune system. ${ }^{2,3}$

Beta-interferon and glatiramer acetate, the current standard first-line treatments of MS, reduce relapse frequency by around $30 \% .^{4-7}$ Natalizumab, ${ }^{8,9}$ and more recently fingolimod, ${ }^{10,11}$ have been introduced for patients with highly active disease; they are more efficacious and have a more concerning safety profile than beta-interferon and glatiramer acetate. Against this background, alemtuzumab has emerged as a potential new therapy in MS, with licensing in Europe and the United States expected in 2013. Here we review its history and the evidence for its efficacy.

\section{The history and pharmacodynamics of alemtuzumab}

Emerging from Cambridge pathology labs in 1983 (and thus originally known as Campath $^{\circledR}$ [now owned by Genzyme Corporation, Cambridge, MA, USA]), alemtuzumab was the first therapeutic humanized monoclonal antibody to be made (hence, "Campath-1H"). It is currently a licensed first-line treatment for B-cell chronic lymphocytic leukemia and has shown efficacy off-license in treating rheumatoid arthritis, ${ }^{12}$ systemic vasculitis, ${ }^{13,14}$ autoimmune cytopenias, ${ }^{15}$ chronic inflammatory demyelinating polyneuropathy, ${ }^{16}$ and uveitis $;{ }^{17}$ there is also growing evidence for its role in preventing chronic rejection and graft-versus-host disease following transplantation. ${ }^{18,19}$

Alemtuzumab is directed against the CD52 antigen, a surface glycoprotein of unknown function expressed on all mature lymphocytes, but not on hematological precursors. Treatment causes antibody-dependent cell-mediated lysis, producing a profound lymphopenia from which B-cells rapidly recover while CD4+ T-helper cells take up to 5 years to reach pretreatment levels. ${ }^{20}$ We speculate that the therapeutic mechanism of action of alemtuzumab lies not in the depletion of effector T-cells, but rather in the complex reorganization of the immune repertoire that follows homeostatic reconstitution of lymphocytes after depletion. $^{21}$

\section{Clinical trials of alemtuzumab}

A literature search on November 20, 2012 revealed six trials reporting the efficacy of alemtuzumab in RRMS (Tables 1 and 2). Four of these have been coordinated from Cambridge where all patients continue to be followed-up within an extension study. ${ }^{22-25}$

\section{Open-label experience of using alemtuzumab}

In 1991, Alastair Compston instigated alemtuzumab's first clinical trial in 58 patients with MS. ${ }^{24}$ The initial cohort was comprised of 36 patients with secondary progressive MS; despite a reduction in annualized relapse rate (ARR) from $0.7-0.001(P<0.001)$ and an absence of new lesions on magnetic resonance imaging (MRI), patients relentlessly accrued clinical disability and radiological evidence of cerebral atrophy. To test whether administration earlier in the disease might halt progression of disability, alemtuzumab was administered to a second cohort comprising 22 patients with RRMS in whom licensed treatment had failed or a high early relapse rate predicted a poor prognosis; the high baseline mean annualized relapse rate (2.2 relapses/patient/year) and disability (4.8 Expanded Disability Status Scale (EDSS) points ${ }^{26}$ ) reflect an aggressive and rapidly progressing cohort. Patients received five consecutive daily doses of $20 \mathrm{mg}$ intravenous alemtuzumab and were followed up for a mean of 29 months; most received methylprednisolone pretreatment to ameliorate the associated cytokine release syndrome and $19(86 \%)$ agreed to a second 3-day course after 1 year. Of these, three required a third course following a relapse. Effect on relapse rate was measured by comparing change in the ARR, while disability was examined by comparing change in the EDSS score, where $0=$ normal and $10=$ dead due to MS. After 2 years, the ARR and EDSS score had improved by $94 \%(P<0.001)$ and 1.2 points, respectively (Table 1). This contrasting effect on disability in RRMS was hypothesized to reflect an early rescue of neurons from an inflammatory environment before axonal damage (neurodegeneration) occurred; thus the notion of an early window of opportunity to slow disability arose.

Buoyed by these results, Hirst et $\mathrm{al}^{27}$ administered alemtuzumab to a cohort of 39 patients with RRMS and a poor prognosis. Thirty-two patients $(82 \%)$ were drug-naïve, and the remainder had failed to respond to first-line diseasemodifying therapy. After 21 months, the ARR had decreased by $92 \%(P<0.0001)$ and the mean EDSS score had improved by 0.36 points compared to baseline. Of the 34 patients completing follow-up EDSS assessment (87\%), the 12 patients with an unstable baseline EDSS score averaged an improvement of 1.5 points, while the remaining 22 averaged a deterioration of 0.2 points, providing evidence for alemtuzumab's particular benefit in active disease. One or more early infusion reactions occurred in 22 patients $(56 \%)$; 12 (31\%) developed biochemical evidence, and three (8\%) 


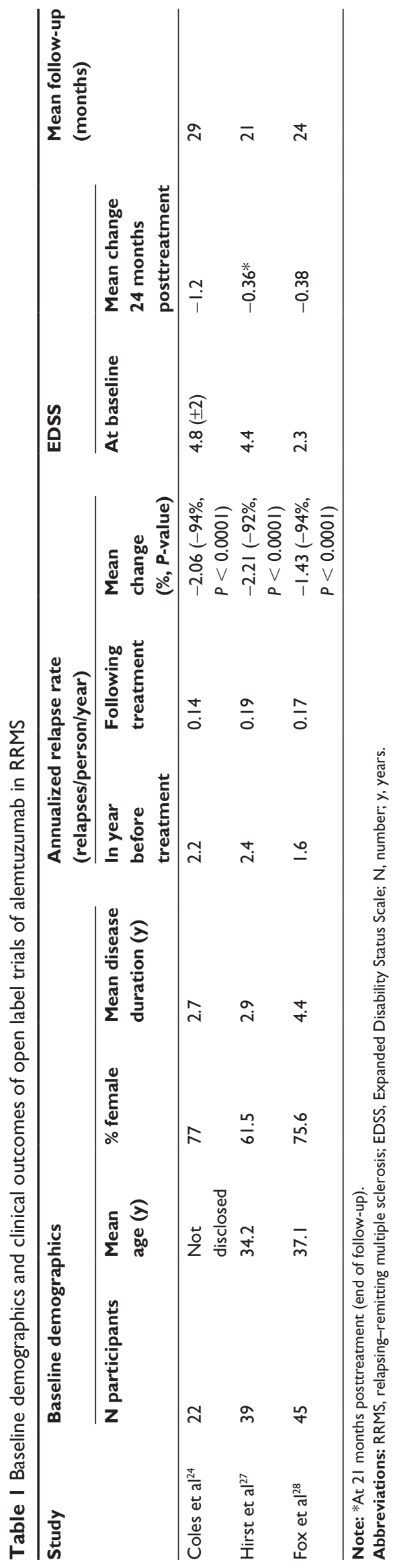

exhibited clinical evidence of autoimmune dysfunction; of the latter, two patients developed thyroid disease and one developed autoimmune skin disease. Seven (18\%) of the patients experienced one or more infections during follow-up, and two (5\%) developed stage 3 cervical intraepithelial neoplasia (all successfully treated).

Fox et $\mathrm{al}^{28}$ recently published the results of a noncontrolled study in 45 patients with RRMS who had relapsed despite interferon therapy. After 2 years, the ARR had decreased by $94 \%(P<0.0001)$ with a mean improvement of 0.38 EDSS points $(P=0.0542)$. Five patients experienced thyroid disorders and single cases of pulmonary embolism; in addition, deep venous thrombosis and pneumonia with neutropenia were reported.

\section{CAMMS223}

In 2008 the results of the CAMMS223 trial were published. ${ }^{23}$ This Phase II rater-blinded trial randomized 334 treatmentnaïve patients from 49 European and American centers with RRMS to receive annual cycles of alemtuzumab or subcutaneous interferon beta-1a $44 \mu \mathrm{g}$, three times per week. Alemtuzumab was given at two different daily doses (12 mg or $24 \mathrm{mg}$ ) giving three treatment groups in total, all of which received methylprednisolone to coincide with the infusion cycles of premedication required by the alemtuzumab groups. Participants had early and active disease (less than 3 years of disease duration, a baseline EDSS score of 3 or less, and at least two relapses in the previous 2 years). The primary outcomes were time to sustained accumulation of disability and change in relapse frequency; secondary outcomes included the proportion of patients who did not have a relapse and radiographic changes (specifically changes in lesion load and brain volume). The study was adequately powered for these outcomes. As there were no significant differences between the two alemtuzumab dose groups' results, their data was pooled.

Compared to interferon beta- $1 \mathrm{a}$, alemtuzumab reduced the risk of sustained disability by $71 \%$ (hazard ratio (HR) 0.29 (95\% confidence interval (CI): $0.16-0.54 ; P<0.001)$ ). After 36 months the mean disability (EDSS) score in the alemtuzumab group had improved from 1.9 to 1.51 (95\% CI 0.23-0.55), while that of the interferon group worsened from 1.9 to 2.28 (95\% CI $0.13-0.63)$, representing a net advantage to alemtuzumab of 0.77 EDSS points $(95 \%$ CI $0.48-1.06, P<0.001)$. This reduction would change patients" categorization as "minimal disability" to "abnormal neurological signs without disability." ${ }^{26}$ At 36 months the relapse rates were 0.11 (alemtuzumab group) and 0.36 
(interferon beta-1a group); thus alemtuzumab decreased the relapse rate by $74 \%$ more than interferon (HR $0.26,95 \%$ CI $0.16-0.41, P<0.001)$. After 36 months, $80 \%$ of patients taking alemtuzumab and $52 \%$ of those taking interferon beta-1a remained relapse-free.

The effects on clinical parameters must be weighed up with the side effects, most notably autoimmunity (see the Discussion section). Alemtuzumab administration was suspended 3 years into the trial, owing to immune thrombocytopenic purpura (ITP) affecting three patients (one fatally due to intracranial hemorrhage). Despite $99 \%$ and only $28 \%$ receiving their second and third cycles of alemtuzumab, respectively, efficacy was maintained at 36 months, highlighting the sustained therapeutic effects. A total of $59 \%$ of the interferon beta-1a group completed the 36-month study compared to $83 \%$ of those receiving alemtuzumab.

The 5-year follow-up data on a subset of patients from CAMMS223 have recently been published. ${ }^{29}$ Forty-two (38\%) of the interferon beta-1 a group continued on that treatment or on other disease-modifying therapy, whereas 141 (65\%) of the alemtuzumab group received no further therapy, except for a small number who received other treatments, and four patients who had additional alemtuzumab cycles. Attrition bias was addressed through sensitivity analyses using inverse probability weighting. In those randomized to alemtuzumab, the baseline demographics, baseline disease characteristics, and ARR were comparable between those who did not participate in the extension phase to those who did; however, the latter had a lower rate of sustained accumulation of disability during the initial study phase. Those randomized to interferon beta-1a that participated in the extension phase had a significantly lower ARR and sustained accumulation of disability than those who elected not to continue. The results confirm that alemtuzumab's superior efficacy over interferon beta-1a extends to at least 5 years. The ARR was 69\% lower in the alemtuzumab group ( 0.11 versus $0.35, P<0.0001)$. Despite only eight patients $(5.7 \%)$ receiving further alemtuzumab in this timeframe, the ARR from year 3 to 5 was still lower with alemtuzumab $(0.14$ versus 0.28$)$, though this narrowly missed statistical significance $(P=0.072)$. After 5 years, significantly more of the alemtuzumab group were relapse-free ( $72 \%$ versus $41 \%$ ). Sustained accumulation of disability was $72 \%$ lower with alemtuzumab $(P<0.0001)$; after sensitivity analysis for informative dropout, this figure reduced to $67 \%$ ( $P<0.0001)$. A subanalysis of those only receiving two doses of alemtuzumab (ie, at randomization and 1 year) had little effect on these figures, highlighting the maintenance of efficacy 4 years after treatment; this also suggests that clinical benefit is still being derived in the presence of (modified) peripheral T-cell reconstitution. ${ }^{30}$ The mean change in EDSS score from baseline to 5 years was +0.46 in the interferon beta-1a group and -0.30 in the alemtuzumab group, yielding a net advantage of 0.76 EDSS points with alemtuzumab. A subset analysis of the CAMMS223 data found that relapse and disability outcomes were improved with alemtuzumab compared to interferon beta-1a regardless of age, sex, geographic region, MRI-T1 brain volume, MRI-T2 lesion volume, disease duration, number of previous relapses within 2 years, and EDSS score. ${ }^{31}$

\section{CARE-MSI and CARE-MS2}

In two recent Phase III active comparator trials (Comparison of Alemtuzumab and Rebif Efficacy in MS, CARE-MS), treatment naïve-patients (in CARE-MS1) 22 and those who had recently relapsed on standard disease-modifying therapy (in CARE-MS2) ${ }^{25}$ were randomized to either subcutaneous interferon beta-1a or intravenous alemtuzumab and were followed up for 2 years. Coprimary outcomes in both studies were relapse rate and time to 6-month sustained accumulation of disability. By comparison to CAMMS223, the inclusion criteria were broader.

\section{CARE-MSI}

A total of 581 patients were randomized to receive either alemtuzumab (12 $\mathrm{mg}$ for 5 days at baseline and for 3 days at 12 months) or interferon beta-1a ( $44 \mu \mathrm{g}$ three times per week) in a $2: 1$ ratio, respectively. Ten $(2.6 \%)$ of those in the alemtuzumab group and eight $(4.1 \%)$ of those in the interferon beta-1a group did not receive the drug (mainly through withdrawing consent). The population represented early, active, and untreated RRMS; inclusion required at least two relapses in the previous 2 years (and one in the preceding year), disease duration not exceeding 5 years, and an EDSS score of 3 or less (Table 2). A total of $96 \%$ of those receiving alemtuzumab and $88 \%$ of those receiving interferon beta-1a completed the study (but all were included in the primary analysis).

After 2 years, $22 \%$ of patients in the alemtuzumab group and $40 \%$ of patients in the interferon beta-1a group had relapsed, generating a HR for relapse with alemtuzumab of 0.45 (95\% CI: $0.32-0.63, P<0.0001)$ versus interferon beta-1a. Unlike previous studies, there was no statistically significant difference in the accumulation of disability; $8 \%$ of the alemtuzumab group and $11 \%$ of the interferon beta-1a group accumulated disability (HR 0.7, 95\% CI 0.4-1.23, $P=0.22)$; the actual change in mean EDSS score was -0.14 


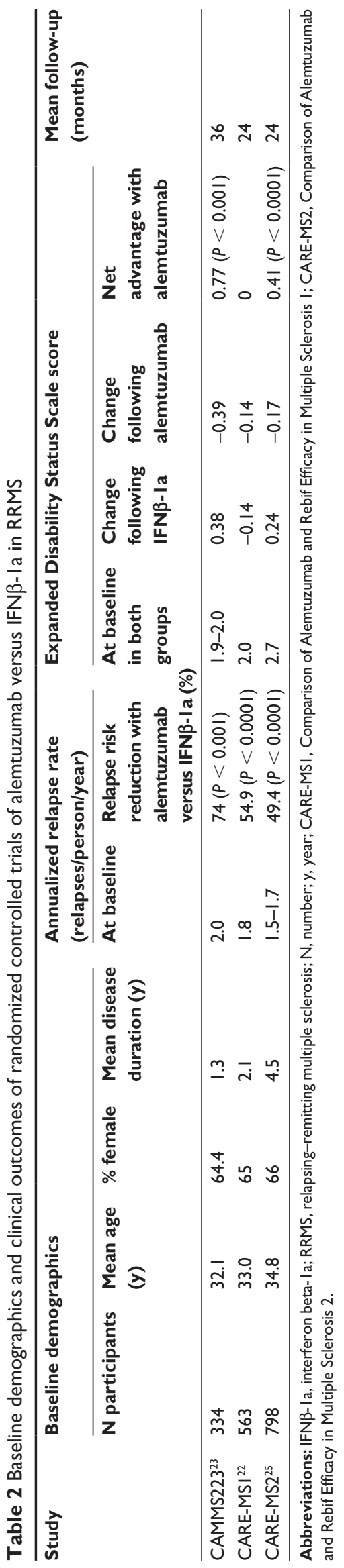

points in both groups. One possible explanation for the lack of significant disability difference is that the proportion of interferon-treated patients achieving the disability endpoint was much lower than the $20 \%$ expected, rendering the trial underpowered. The reduction in T2 hyperintense lesion volume on MRI was not significantly greater with alemtuzumab either $(-9.3 \%$ versus $-6.5 \%, P=0.31)$. Use of this as the standard measure of lesion acquisition was derived from placebo-controlled trials; that interferon is known to reduce T2 lesion volume in RRMS questions the suitability of this measure and probably accounts for the apparent lack of alemtuzumab's greater effect in this parameter., ${ }^{4,5}$ However, there were significantly fewer patients in the alemtuzumab group with new or enlarging T2 hyperintense lesions (48\% versus $58 \%, P=0.04$ ) and gadolinium-enhancing (ie, less than 6 -week-old) T1 lesions ( $7 \%$ versus $19 \%, P<0.0001$ ). A significantly lower rate of brain volume loss (atrophy) was noted with alemtuzumab compared to interferon beta-1a, as measured by change in brain parenchymal fraction $(-0.867 \%$ versus $-1.488 \%, P<0.0001)$. There was a higher MS functional composite increase in the alemtuzumab group compared to the interferon group ( 0.15 versus $0.07, P=0.01)$. After 2 years, $74 \%$ of patients were clinically disease-free and $39 \%$ were clinically and radiologically disease-free in the alemtuzumab group (compared to $56 \%$ and $27 \%$, respectively, in the interferon beta-1a group). The odds ratios for the clinical and clinical-and-radiological disease-free survival were thus 2.36 and 1.75 , respectively (both $P<0.01$ ), compared to interferon beta-1a.

\section{CARE-MS2}

Patients with active disease despite first-line treatment pose a therapeutic challenge with little robust evidence to guide physicians. Following encouraging efficacy results in subgroup analyses of previous trials, ${ }^{24,27}$ alemtuzumab was again compared to interferon beta-1a in 840 such patients. Inclusion required disease duration of 10 years or less, a baseline EDSS score of 5 or less, two relapses within the last 2 years (and at least one in the previous year), and at least one relapse despite 6 months or more of interferon beta or glatiramer treatment. In all, $90 \%$ of the alemtuzumab group and $84 \%$ of the interferon beta-1a group had previously received interferon beta-1a or interferon beta- $1 \mathrm{~b}, 34 \%$ of each group had received glatiramer, and 3\%-4\% had been administered natalizumab (all $P>0.05$ ).

After 2 years, $34 \%$ of patients taking alemtuzumab and $53 \%$ of those taking interferon beta- 1 a had relapsed, generating a hazard ratio for relapse with alemtuzumab of 0.51 
(95\% CI: $0.39-0.65, P<0.0001)$ compared to interferon beta-1a. These numbers translate to ARRs of 0.26 for alemtuzumab and 0.52 for interferon beta- 1 a (refer to the corresponding ARRs in the drug naïve patients in the CARE-MS1 study of 0.18 and 0.39 , respectively). In contrast to CAREMS1, a significantly lower percentage of patients accrued sustained disability over 6 months with alemtuzumab compared to interferon beta-1a (12.7\% versus $21.3 \%$, HR 0.58 (95\% CI 0.38-0.87, $P=0.0084)$ ). Further, the EDSS score of those taking interferon beta-1a worsened by 0.24 points compared to an improvement by 0.17 points with alemtuzumab $(P<0.0001)$. The MRI findings followed a similar trend to CARE-MS1: the median change in T2-hyperintense lesion volume was comparable between groups $(-1.23 \%$, versus $-1.27 \%, P=0.14$ ), but fewer patients taking alemtuzumab had new or enlarging T2-hyperintense lesions (46\% versus $68 \%, P<0.0001)$ and gadolinium-enhancing T1 lesions ( $9 \%$ versus $23 \%, P<0.0001$ ); brain atrophy was significantly less with alemtuzumab $(-0.61 \%$ versus $0.81 \%$, $P<0.01$ ). After 2 years, $60 \%$ of patients were clinically disease-free and $32 \%$ were clinically and radiologically disease-free in the alemtuzumab group (compared to $41 \%$ and $14 \%$, respectively, in the interferon group).

The number of patients who would need to be treated with alemtuzumab instead of interferon beta-1a to enable one patient to be clinically free of MS at 2 years was 5.6 (in CARE-MS1) and 5.3 (in CARE-MS2); the corresponding numbers to achieve clinical and radiological freedom at 2 years were 8.3 and 5.6 , respectively. ${ }^{22,25}$

\section{Safety}

The adverse event profile of alemtuzumab may broadly be divided into infusion reactions, infections, and autoimmunity. Within active comparator trials, ${ }^{22,23,25}$ adverse events were more common in the alemtuzumab groups than the interferon beta-1a groups (7.2-8.66 versus 4.9-5.7 events per person-year) and serious adverse events followed a similar though slightly less demarcated trend (0.13-2.0 versus $0.09-0.3$ events per person year). Drug discontinuation due to side effects was persistently lower in patients taking alemtuzumab compared to those taking interferon beta- $1 \mathrm{a}$ $(1 \%-3.3 \%$ versus $6 \%-12.1 \%)$. In the CAMMS223 trial, one patient in the alemtuzumab group died from preexisting cardiovascular disease, and one patient died from a brain hemorrhage secondary to ITP; no patients on interferon died in the study period. Within CARE-MS1 and CARE-MS2, no patients taking interferon beta-1a died compared to four taking alemtuzumab (two in each study); one patient in each group died following automobile accidents. The remaining patient in CARE-MS1 died from sepsis after the study period, while in CARE MS2, a patient died of respiratory complications due to severe disability from a brainstem relapse some months before.

A total of $90 \%-99 \%$ of patients taking alemtuzumab experienced infusion reactions, which were largely mild and consisted of headache, rash, nausea, and pyrexia. Infections were predominantly of the upper respiratory and urinary tracts; an increased frequency of superficial herpes infections was reduced by prophylactic administration of acyclovir (Table 3).

\section{Autoimmunity after alemtuzumab}

The principal adverse effect of alemtuzumab is autoimmunity (Table 4), which has been comprehensively reviewed elsewhere. ${ }^{32}$ Such autoimmunity is thought to arise from faulty peripheral reconstitution of the lymphocyte repertoire in response to alemtuzumab treatment in individuals rendered susceptible by high interleukin (IL)-21 secretion. ${ }^{33}$ Having shown that high pretreatment serum IL-21 predisposes patients to autoimmunity, we are currently exploring this and other cytokines as predictive biomarkers that could be used to individualize risks for patient counseling before treatment and the intensity of monitoring after treatment.

Within Phase III trials, $17 \%-18 \%$ of patients randomized to alemtuzumab experienced thyroid dysfunction (compared to $5 \%-6 \%$ of those taking interferon beta- $1 \mathrm{a}),{ }^{22,25}$ including hypo- and hyperthyroidism, goiter, and thyroiditis; all cases responded to conventional treatment. In all,

Table 3 Summary of infective and malignant adverse events from active comparator trials

\begin{tabular}{|c|c|c|c|c|c|c|}
\hline & \multicolumn{2}{|c|}{ Any infection } & \multicolumn{2}{|c|}{ Serious infection } & \multicolumn{2}{|l|}{ Cancer } \\
\hline & $\begin{array}{l}\text { IFN } \beta \text { - I a } \\
\mathbf{N}(\%)\end{array}$ & $\begin{array}{l}\text { Alemtuzumab } \\
\mathbf{N}(\%)\end{array}$ & $\begin{array}{l}\text { IFN } \beta \text { - I a } \\
\mathbf{N}(\%)\end{array}$ & $\begin{array}{l}\text { Alemtuzumab } \\
\mathbf{N}(\%)\end{array}$ & $\begin{array}{l}\text { IFN } \beta \text { - I a } \\
\mathbf{N}(\%)\end{array}$ & $\begin{array}{l}\text { Alemtuzumab } \\
\mathbf{N}(\%)\end{array}$ \\
\hline CAMMS $223^{23}$ & $50(46.7)$ & $142(65.7)$ & $2(1.9)$ & $9(4.2)$ & $I(I)$ & $3(1)$ \\
\hline CARE-MSI ${ }^{22}$ & $85(45)$ & $253(67)$ & $2(1)$ & $7(2)$ & 0 & $2(1)$ \\
\hline CARE-MS2 $2^{25}$ & $134(66)$ & $468(79)$ & $3(1)$ & $22(4)$ & $2(I)$ & $5(1)$ \\
\hline
\end{tabular}

Abbreviations: IFN $\beta$-la, interferon beta-la; N, number; CARE-MSI, Comparison of Alemtuzumab and Rebif Efficacy in Multiple Sclerosis I; CARE-MS2, Comparison of Alemtuzumab and Rebif Efficacy in Multiple Sclerosis 2. 
Table 4 Autoimmune adverse events in active comparator trials

\begin{tabular}{|c|c|c|c|c|}
\hline & \multicolumn{2}{|c|}{ Thyroid-associated autoimmune event } & \multicolumn{2}{|c|}{ Immune thrombocytopenic purpura } \\
\hline & IFN $\beta$ - I a N (\%) & Alemtuzumab N (\%) & IFN $\beta$ - I a N (\%) & Alemtuzumab N (\%) \\
\hline CAMMS22333,a & $3(2.8)$ & $49(22.7)$ & $\mathrm{I}(0.9)$ & $6(2.8)$ \\
\hline CARE-MSI 22,a & $12(6)$ & $68(18)$ & 0 & $3(1)$ \\
\hline CARE-MS2 $2^{25, a}$ & $10(5)$ & $100(17)$ & 0 & $5(1)$ \\
\hline
\end{tabular}

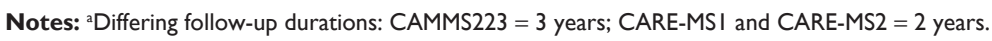

Abbreviations: IFN $\beta$-Ia, interferon beta-Ia; N, number; CARE-MSI, Comparison of Alemtuzumab and Rebif Efficacy in Multiple Sclerosis I; CARE-MS2, Comparison of Alemtuzumab and Rebif Efficacy in Multiple Sclerosis 2.

$1 \%$ experienced ITP, $;^{22,25}$ one patient required splenectomy. A rigorous monitoring system for adverse events was instigated during and after the CAMMS223 trial including monthly patient questionnaires and full blood counts, thyroid function testine every 3 months, and participant and educator education about recognizing adverse events and instigating appropriate management.

\section{Discussion}

Three open-label trials, one Phase II trial and two Phase III trials against an active comparator, have verified alemtuzumab's efficacy in reducing relapse frequency, reducing the risk of sustaining disability, partially reversing disability, and improving radiological markers in relapsing remitting MS.

A comparison of reduction in relapse rates and EDSS scores between trials following alemtuzumab reveals no obvious trends reflecting differing inclusion criteria (Tables 1 and 2); the absence of any correlation with baseline relapse rate, EDSS score, or disease duration is in keeping with the subgroup analyses that failed to identify characteristics predicting the most benefit. ${ }^{31}$ There is some noncontrolled evidence suggesting greater efficacy in patients with unstable disease at baseline, ${ }^{27}$ however, sensitivity analyses in active comparator trials found that an active scan at baseline (with gadolinium-enhancing lesions) failed to predict treatment response or degree of improvement. ${ }^{22,23,25}$ Most trials were undertaken in untreated, early, active, and aggressive RRMS, ${ }^{22-24,27}$ and thus generalization should be limited to such populations; the baseline demographics (young, predominantly female patients) correspond to the disease epidemiology increasing the results' applicability. One (adequately powered) study supports the use of alemtuzumab in patients with longer disease duration (mean of 4.5 years) and previous unsuccessful treatment. ${ }^{25}$

No trial has compared alemtuzumab with natalizumab, a monthly humanized monoclonal antibody licensed for highly active RRMS. The differing interventions of the two major natalizumab trials make even indirect comparisons difficult; the AFFIRM study compared natalizumab to placebo, ${ }^{8}$ while the SENTINEL trial compared natalizumab combined with interferon beta-1a to placebo combined with interferon beta-1a in patients who had relapsed on standard therapy. ${ }^{9}$

In considering the place of alemtuzumab in the landscape of MS treatments, its convenience of administration and unequaled effects on relapse frequency and disability accumulation must be weighed against the high frequency of potentially serious, but treatable, side effects. The licensing authorities will define its target population; until then, our proposal would be that alemtuzumab is suitable for people with relapsing remitting MS, within 10 years of disease onset, who have high relapse frequency off treatment, or any relapse activity whilst on disease-modifying therapy. Two developments would improve access for people with MS to alemtuzumab: a robust predictive biomarker of autoimmunity after alemtuzumab (such as IL-21, as above), and/or a method for reducing the risk of autoimmunity (such as is being tested in a current trial of alemtuzumab combined with palifermin, ClinicalTrials. gov number, NCT01712945). The licensing authorities will also define the appropriate safety monitoring regime; at present, we suggest monthly urine and platelet monitoring and quarterly thyroid monitoring for 5 years after last alemtuzumab treatment.

\section{Acknowledgments}

Alasdair Coles is supported by the Cambridge Biomedical Research Center of the National Institute of Health Research.

\section{Disclosure}

The authors report no conflicts of interest in this work. Alasdair Coles has received personal honoraria for consultancies with Genzyme and Merk Serono, and institutional grant support from Genzyme.

\section{References}

1. Trisolini M, Honeycutt A, Wiener J, Lesesne S; for Multiple Sclerosis International Federation. Global Economic Impact of Multiple Sclerosis. London: Multiple Sclerosis International Federation; 2010. 
2. Compston A, Coles A. Multiple sclerosis. Lancet. 2008;372(9648): $1502-1517$.

3. Disanto G, Morahan JM, Barnett MH, Giovannoni G, Ramagopalan SV. The evidence for a role of B cells in multiple sclerosis. Neurology. 2012;78(11):823-832.

4. Interferon beta- $1 \mathrm{~b}$ in the treatment of multiple sclerosis: final outcome of the randomized controlled trial. The IFNB Multiple Sclerosis Study Group and The University of British Columbia MS/MRI Analysis Group. Neurology. 1995;45(7):1277-1285.

5. Randomised double-blind placebo-controlled study of interferon beta-1a in relapsing/remitting multiple sclerosis. PRISMS (Prevention of Relapses and Disability by Interferon beta-1a Subcutaneously in Multiple Sclerosis) Study Group. Lancet. 1998;352(9139):1498-1504.

6. Jacobs LD, Cookfair DL, Rudick RA, et al. Intramuscular interferon beta-1a for disease progression in relapsing multiple sclerosis. The Multiple Sclerosis Collaborative Research Group (MSCRG). Ann Neurol. 1996;39(3):285-294.

7. Johnson KP, Brooks BR, Cohen JA, et al. Copolymer 1 reduces relapse rate and improves disability in relapsing-remitting multiple sclerosis: results of a phase III multicenter, double-blind placebo-controlled trial. The Copolymer 1 Multiple Sclerosis Study Group. Neurology. 1995;45(7):1268-1276.

8. Polman CH, O'Connor PW, Havrdova E, et al; for AFFIRM Investigators. A randomized, placebo-controlled trial of natalizumab for relapsing multiple sclerosis. $N$ Engl J Med. 2006;354(9):899-910.

9. Rudick RA, Stuart WH, Calabresi PA, et al; for SENTINEL Investigators. Natalizumab plus interferon beta-1a for relapsing multiple sclerosis. N Engl J Med. 2006;354(9):911-923.

10. Cohen JA, Barkhof F, Comi G, et al; for TRANSFORMS Study Group. Oral fingolimod or intramuscular interferon for relapsing multiple sclerosis. N Engl J Med. 2010;362(5):402-415.

11. Kappos L, Radue EW, O'Connor P, et al; for FREEDOMS Study Group. A placebo-controlled trial of oral fingolimod in relapsing multiple sclerosis. N Engl J Med. 2010;362(5):387-401.

12. Schnitzer TJ, Yocum DE, Michalska M, et al. Subcutaneous administration of CAMPATH-1H: clinical and biological outcomes. J Rheumatol. 1997;24(6):1031-1036.

13. Lockwood CM, Thiru S, Isaacs JD, Hale G, Waldmann H. Long-term remission of intractable systemic vasculitis with monoclonal antibody therapy. Lancet. 1993;341(8861):1620-1622.

14. Lockwood CM, Thiru S, Stewart S, et al. Treatment of refractory Wegener's granulomatosis with humanized monoclonal antibodies. QJM. 1996;89(12):903-912.

15. Lim SH, Hale G, Marcus RE, Waldmann H, Baglin TP. CAMPATH-1 monoclonal antibody therapy in severe refractory autoimmune thrombocytopenic purpura. Br J Haematol. 1993;84(3):542-544.

16. Marsh EA, Hirst CL, Llewelyn JG, et al. Alemtuzumab in the treatment of IVIG-dependent chronic inflammatory demyelinating polyneuropathy. J Neurol. 2010;257(6):913-919.

17. Isaacs JD, Hale G, Waldmann $\mathrm{H}$, et al. Monoclonal antibody therapy of chronic intraocular inflammation using Campath-1H. Br J Ophthalmol. 1995;79(11):1054-1055.
18. Zhou J, Ju WQ, He XS, et al. Effect of Campath-1H induction on immunosuppression in small intestine transplantation. Zhonghua Wei Chang Wai Ke Za Zhi. 2011;14(3):199-201. Chinese.

19. Barth RN, Janus CA, Lillesand CA, et al. Outcomes at 3 years of a prospective pilot study of Campath-1H and sirolimus immunosuppression for renal transplantation. Transpl Int. 2006;19(11):885-892.

20. Hill-Cawthorne GA, Button T, Tuohy O, et al. Long term lymphocyte reconstitution after alemtuzumab treatment of multiple sclerosis. J Neurol Neurosurg Psychiatry. 2012;83(3):298-304.

21. Cox AL, Thompson SA, Jones JL, et al. Lymphocyte homeostasis following therapeutic lymphocyte depletion in multiple sclerosis. Eur $J$ Immunol. 2005;35(11):3332-3342.

22. Cohen JA, Coles AJ, Arnold DL, et al; for CARE-MS I investigators. Alemtuzumab versus interferon beta $1 \mathrm{a}$ as first-line treatment for patients with relapsing-remitting multiple sclerosis: a randomised controlled phase 3 trial. Lancet. 2012;380(9856):1819-1828.

23. Coles AJ, Compston DA, Selmaj KW, et al; for CAMMS223 Investigators. Alemtuzumab vs interferon beta-1a in early multiple sclerosis. N Engl J Med. 2008;359(17):1786-1801.

24. Coles AJ, Cox A, Le Page E, et al. The window of therapeutic opportunity in multiple sclerosis: evidence from monoclonal antibody therapy. J Neurol. 2006;253(1):98-108.

25. Coles AJ, Twyman CL, Arnold DL, et al; for CARE-MS II investigators. Alemtuzumab for patients with relapsing multiple sclerosis after disease-modifying therapy: a randomised controlled phase 3 trial. Lancet. 2012;380(9856):1829-1839.

26. Kurtzke JF. Rating neurologic impairment in multiple sclerosis: an expanded disability status scale (EDSS). Neurology. 1983; 33(11):1444-1452.

27. Hirst CL, Pace A, Pickersgill TP, et al. Campath 1-H treatment in patients with aggressive relapsing remitting multiple sclerosis. J Neurol. 2008;255(2):231-238.

28. Fox EJ, Sullivan HC, Gazda SK, et al. A single-arm, open-label study of alemtuzumab in treatment-refractory patients with multiple sclerosis. Eur J Neurol. 2012;19(2):307-311.

29. Coles AJ, Fox E, Vladic A, et al. Alemtuzumab more effective than interferon $\beta$-1a at 5-year follow-up of CAMMS223 clinical trial. Neurology. 2012;78(14):1069-1078.

30. Thompson SA, Jones JL, Cox AL, Compston DA, Coles AJ. B-cell reconstitution and BAFF after alemtuzumab (Campath-1H) treatment of multiple sclerosis. J Clin Immunol. 2010;30(1):99-105.

31. Coles AJ, Fox E, Vladic A, et al. Alemtuzumab versus interferon $\beta$ - 1 a in early relapsing-remitting multiple sclerosis: post-hoc and subset analyses of clinical efficacy outcomes. Lancet Neurol. 2011;10(4):338-348.

32. Costelloe L, Jones J, Coles A. Secondary autoimmune diseases following alemtuzumab therapy for multiple sclerosis. Expert Rev Neurother. 2012;12(3):335-341.

33. Jones JL, Phuah CL, Cox AL, et al. IL-21 drives secondary autoimmunity in patients with multiple sclerosis, following therapeutic lymphocyte depletion with alemtuzumab (Campath-1H). J Clin Invest. 2009;119(7):2052-2061.
Drug Design, Development and Therapy

\section{Publish your work in this journal}

Drug Design, Development and Therapy is an international, peerreviewed open-access journal that spans the spectrum of drug design and development through to clinical applications. Clinical outcomes, patient safety, and programs for the development and effective, safe, and sustained use of medicines are a feature of the journal, which

\section{Dovepress}

has also been accepted for indexing on PubMed Central. The manuscript management system is completely online and includes a very quick and fair peer-review system, which is all easy to use. Visit $\mathrm{http}: / /$ www.dovepress.com/testimonials.php to read real quotes from published authors. 